\title{
EDGE OF THE WEDGE THEORY IN INVOLUTIVE STRUCTURES*
}

\author{
ZIAD ADWAN $^{\dagger}$ AND SHIFERAW BERHANU ${ }^{\dagger}$
}

\begin{abstract}
This paper describes the $C^{\infty}$ wave-front set of the boundary values of approximate solutions in wedges $\mathcal{W}$ of involutive structures $(M, \mathcal{V})$ that are not necessarily locally integrable. It is shown that the $C^{\infty}$ wave-front set of the boundary value is contained in the polar of a certain cone $\Gamma^{T}(\mathcal{W})$ contained in $\Re \mathcal{V} \cap T X$ where $X$ is a maximally real edge of $\mathcal{W}$. A converse result is also established.
\end{abstract}

Key words. Involutive, Maximally real, Wedge, Edge, Wave-front, approximate solution

AMS subject classifications. Primary 35F15, 35B30, 42B30; Secondary 42A38, 30E25

1. Introduction. Let $M$ be a $C^{\infty}$ manifold and $\mathcal{V} \subseteq \mathbb{C} T M$ a subbundle of rank $n$ which is involutive, that is, the bracket of two smooth sections of $\mathcal{V}$ is also a section of $\mathcal{V}$. We will refer to the pair $(M, \mathcal{V})$ as an involutive structure. The involutive structure $(M, \mathcal{V})$ is called locally integrable if the orthogonal of $\mathcal{V}$ in $\mathbb{C} T^{*} M$ is locally generated by exact forms. In $[\mathrm{EG}]$ assuming that $(M, \mathcal{V})$ is locally integrable, the authors proved some microlocal regularity results for a distribution $u$ on certain submanifolds $E$ of $M$ where $u$ arises as the boundary value of a solution on a wedge $\mathcal{W}$ in $M$ with edge $E$. These results were expressed in terms of the hypo-analytic wave-front set developed in $[\mathrm{BCT}]$. In this article we prove some analogous results in the setting of involutive structures that are not necessarily locally integrable, and for boundary values of approximate solutions (Definition 2.4) in wedges.

In section 2 we summarize some of the notions from [EG] that we need to state our main results, Theorems 3.1 and 3.2. Section 3 is devoted to the proofs of these results. Finally, in section 4 we present a sufficient condition for the existence of boundary values that is used in the proof of Theorem 3.2.

2. Preliminaries. In this section we will briefly recall some of the notions and results we will need about involutive structures. The reader is referred to [EG] for more details.

We assume $(M, \mathcal{V})$ is an involutive structure and the fiber dimension of $\mathcal{V}$ equals $n$. A distribution $f$ on $M$ is called a solution if $L f=0$ for all smooth sections $L$ of $\mathcal{V}$. A real cotangent vector $\sigma \in T_{p}^{*} M$ is said to be characteristic for the involutive structure $(M, \mathcal{V})$ if $\sigma(L)=0$ for all $L \in \mathcal{V}_{p}$ and we let

$$
T_{p}^{0}=\left\{\sigma \in T_{p}^{*} M: \sigma \text { is characteristic for }(M, \mathcal{V})\right\} .
$$

Even when $\mathcal{V}$ is a line bundle, the dimension of $T_{p}^{0}$ may not be constant as $p$ varies. However, when $\mathcal{V}$ is a $\mathrm{CR}$ structure, that is, $\mathcal{V} \cap \overline{\mathcal{V}}=\{0\}$, then $T^{0}$ is a vector bundle.

Definition 2.1. A smooth submanifold $X$ of $M$ is called maximally real if $\mathbb{C} T_{p} M=\mathcal{V}_{p} \oplus \mathbb{C} T_{p} X$ for each $p \in X$.

If $X$ is a maximally real submanifold and $p \in X$, define

$$
\mathcal{V}_{p}^{X}=\left\{L \in \mathcal{V}_{p}: \Re L \in T_{p} X\right\} .
$$

\footnotetext{
*Received July 21, 2006; accepted for publication February 16, 2007.

${ }^{\dagger}$ Department of Mathematics, Temple University, Philadelphia, PA 19122, USA (ziad@temple. edu; berhanu@temple.edu).
} 
We recall the following result from $[\mathrm{EG}]$ which is also valid for a general involutive structure.

Proposition 2.1. (Lemma II.1 in [EG]) $\mathcal{V}^{X}$ is a real subbundle of $\left.\mathcal{V}\right|_{X}$ of rank $n$. The map

$$
\Im:\left.\mathcal{V}\right|_{X} \rightarrow T M
$$

which takes the imaginary part induces an isomorphism

$$
\left.\mathcal{V}^{X} \cong T M\right|_{X} / T X .
$$

Proposition 2.1 shows that when $X$ is maximally real, for $p \in X, \Im$ defines an isomorphism from $\mathcal{V}_{p}^{X}$ to an $n$-dimensional subspace $N_{p}$ of $T_{p} M$ which is a canonical complement to $T_{p} X$ in the sense that

$$
T_{p} M=T_{p} X \oplus N_{p}
$$

DeFINITION 2.2. Let $E$ be a submanifold of $M, \operatorname{dim}_{\mathbb{R}} E=k$. We say an open set $\mathcal{W}$ is a wedge in $M$ at $p \in E$ with edge $E$ if the following holds: there exists a diffeomorphism $F$ of a neighborhood $V$ of 0 in $\mathbb{R}^{N}\left(N=\operatorname{dim}_{\mathbb{R}} M\right)$ onto a neighborhood $U$ of $p$ in $M$ with $F(0)=p$ and a set $B \times \Gamma \subseteq V$ with $B$ a ball centered at $0 \in \mathbb{R}^{k}$ and $\Gamma$ a truncated, open convex cone in $\mathbb{R}^{N-k}$ with vertex at 0 such that

$$
F(B \times \Gamma)=\mathcal{W} \text { and } F(B \times\{0\})=E \cap U .
$$

Definition 2.3. Let $E, \mathcal{W}$ and $p \in E$ be as in the previous definition. The direction wedge $\Gamma_{p}(\mathcal{W}) \subseteq T_{p} M$ is defined as the interior of the set

$$
\left\{c^{\prime}(0) \mid c:[0,1) \rightarrow M \text { is } C^{\infty}, c(0)=p, c(t) \in \mathcal{W} \forall t>0\right\} .
$$

It is easy to see that $\Gamma_{p}(\mathcal{W})$ is a linear wedge in $T_{p} M$ with edge $T_{p} E$. Set

$$
\Gamma(\mathcal{W})=\bigcup_{p \in E} \Gamma_{p}(\mathcal{W})
$$

Suppose $\mathcal{W}$ is a wedge in $M$ with a maximally real edge $X$. As observed in [EG], since $\Gamma_{p}(\mathcal{W})$ is determined by its image in $T_{p} M / T_{p} X$, the isomorphism $\Im$ can be used to define a corresponding wedge in $\mathcal{V}_{p}^{X}$ by setting

$$
\Gamma_{p}^{\mathcal{V}}(\mathcal{W})=\left\{L \in \mathcal{V}_{p}^{X}: \Im L \in \Gamma_{p}(\mathcal{W})\right\}
$$

$\Gamma_{p}^{\mathcal{V}}(\mathcal{W})$ is a linear wedge in $\mathcal{V}_{p}^{X}$ with edge $\{0\}$, that is, it is a cone. Define also

$$
\Gamma_{p}^{T}(\mathcal{W})=\left\{\Re L: L \in \Gamma_{p}^{\mathcal{V}}(\mathcal{W})\right\}
$$

$\Gamma_{p}^{T}(\mathcal{W})$ is an open cone in $\left(\Re \mathcal{V}_{p}\right) \cap T_{p} X$ (see $\left.[\mathrm{EG}]\right)$. Set

$$
\Gamma^{\mathcal{V}}(\mathcal{W})=\bigcup_{p \in X} \Gamma_{p}^{\mathcal{V}}(\mathcal{W}) \text { and } \Gamma^{T}(\mathcal{W})=\bigcup_{p \in X} \Gamma_{p}^{T}(\mathcal{W})
$$


DeFINITION 2.4. Let $\mathcal{W}$ be a wedge in $M$ with edge a maximally real submanifold $X$. We say a distribution $f \in \mathcal{D}^{\prime}(\mathcal{W})$ is an approximate solution if $L f \in L_{\text {loc }}^{1}(\mathcal{W})$ and

$$
L f(p)=O(\operatorname{dist}(p, X))^{l} \quad \forall l=1,2,3, \ldots,
$$

and for all smooth sections $L$ of $\mathcal{V}$.

Let $\mathcal{W}$ and $X$ be as in Definition 2.4, $f \in \mathcal{D}^{\prime}(\mathcal{W})$ and $u \in \mathcal{D}^{\prime}(X)$. Near a point $p \in X$ let $\left(x^{\prime}, x^{\prime \prime}\right) \in B \times \Gamma$ be a coordinate system where $B$ and $\Gamma$ are as in Definition 2.2. We say that $f$ has a boundary value $u$ if at each $p$ and in each such coordinate system, $f$ is a smooth function on $\Gamma$ with values in $\mathcal{D}^{\prime}(B)$, extends continuously to $\Gamma \cup\{0\}$ and equals $u$ at $x^{\prime \prime}=0$.

\section{Main results and proofs.}

TheOREM 3.1. Let $(M, \mathcal{V})$ be an involutive structure, $\operatorname{dim}_{\mathbb{R}} M=m+n$, rank of $\mathcal{V}=n, X \subset M$ a maximally real submanifold, and $\mathcal{W}$ a wedge in $M$ with edge $X$. Suppose that $u \in \mathcal{D}^{\prime}(X)$ is the boundary value of an approximate solution $f \in \mathcal{D}^{\prime}(\mathcal{W})$. Then

$$
W F(u) \subset\left(\Gamma^{T}(\mathcal{W})\right)^{0} .
$$

(Here $W F(u)$ denotes the $C^{\infty}$ wave-front set of $u$ and $\left(\Gamma^{T}(\mathcal{W})\right)^{0}$ denotes the polar of $\Gamma^{T}(\mathcal{W})$ in the cotangent space $\left.T^{*} X\right)$.

Proof. Since $\mathcal{W}$ is a wedge in $M$ with edge $X$, in a neighborhood $\Omega$ of a point $p \in X$, there are coordinates $(x, t)=\left(x_{1}, \ldots, x_{m}, t_{1}, \ldots, t_{n}\right)$ vanishing at $p$ so that in $\Omega$

$$
\begin{gathered}
X=\{(x, 0):|x|<r\}=B_{r}(0), \\
\mathcal{W}=X \times \Gamma \text { for some open convex cone } \Gamma \subset \mathbb{R}_{t}^{n} .
\end{gathered}
$$

Since $X$ is maximally real,

$$
\mathbb{C} T M=\mathbb{C} T X \oplus \mathcal{V}
$$

and so for each $j=1, \ldots, n$, there exists a smooth section $L_{j}$ of $\mathcal{V}$ ( near 0 ) and smooth functions $a_{j k}(x, t), 1 \leq j \leq n, 1 \leq k \leq m$ such that

$$
L_{j}=\frac{\partial}{\partial t_{j}}+\sum_{k=1}^{m} a_{j k}(x, t) \frac{\partial}{\partial x_{k}} \quad(1 \leq j \leq n) .
$$

Observe that the $L_{j}$ 's are linearly independent over $\mathbb{C}$, and so

$$
\mathcal{V}=\operatorname{span}_{\mathbb{C}}\left\{L_{j}: 1 \leq j \leq n\right\}
$$

Let

$$
\left\{Z_{1}(x, t), \ldots, Z_{m}(x, t)\right\}
$$

be smooth functions satisfying the following properties (see [T]): for all $N \in \mathbb{N}$ there exists $C_{N}>0$ such that

$$
\left|L_{j} Z_{l}(x, t)\right| \leq C_{N}|t|^{N}, \text { and } Z_{l}(x, 0)=x_{l}, \text { for } 1 \leq l \leq m
$$


For $l=1, \ldots, m$, and $(x, t) \in \Omega$, we can write

$$
Z_{l}(x, t)=x_{l}+\sum_{s=1}^{n} t_{s} \psi_{l s}(x, t),
$$

where $\psi_{l s}(x, t)=\psi_{l s}^{(1)}(x, t)+i \psi_{l s}^{(2)}(x, t), \psi_{l s}^{(1)}$ and $\psi_{l s}^{(2)}$ real-valued. Set

$$
Z(x, t)=\left(Z_{1}(x, t), \ldots, Z_{m}(x, t)\right) \text {, and } A(x, t)=\left(\psi_{i j}(x, t)\right)_{1 \leq i \leq m, 1 \leq j \leq n} .
$$

Then we can rewrite (3.2) in the matrix form

$$
Z(x, t)=x+A(x, t) t .
$$

From (3.1), for all $1 \leq j \leq n, 1 \leq l \leq m$

$$
-a_{j l}(0,0)=\psi_{l j}(0,0)
$$

Hence, for all $1 \leq j \leq n, 1 \leq l \leq m$

$$
-\Im a_{j l}(0,0)=\psi_{l j}^{(2)}(0,0)
$$

We have:

$$
\mathcal{V}_{0}^{X}=\left\{L \in \mathcal{V}_{0}: \Re L \in T_{0} X\right\}=\operatorname{span}_{\mathbb{R}}\left\{\left.i L_{j}\right|_{0}: 1 \leq j \leq n\right\} .
$$

Indeed, the above span is contained in $\mathcal{V}_{0}^{X}$ and since its dimension over $\mathbb{R}$ is $n$, by Proposition 2.1, it equals $\mathcal{V}_{0}^{X}$. The direction wedge

$$
\Gamma_{0}(\mathcal{W})=\left\{\left.\sum_{j=1}^{m} a_{j} \frac{\partial}{\partial x_{j}}\right|_{0}+\left.\sum_{j=1}^{n} b_{j} \frac{\partial}{\partial t_{j}}\right|_{0}: a \in \mathbb{R}^{m}, b \in \Gamma\right\} \simeq \mathbb{R}^{m} \times \Gamma .
$$

Hence,

$$
\Gamma_{0}^{\mathcal{V}}(\mathcal{W})=\left\{L \in \mathcal{V}_{0}^{X}: \Im L \in \Gamma_{0}(\mathcal{W})\right\}=\left\{\left.\sum_{j=1}^{n} i b_{j} L_{j}\right|_{0}: b \in \Gamma\right\}
$$

and

$$
\begin{aligned}
\Gamma_{0}^{T}(\mathcal{W}) & =\left\{\Re L: L \in \Gamma_{0}^{\mathcal{V}}(\mathcal{W})\right\} \\
& =\left\{\sum_{j=1}^{n} b_{j}\left(\sum_{k=1}^{m}-\left.\Im a_{j k}(0,0) \frac{\partial}{\partial x_{k}}\right|_{0}\right): b \in \Gamma\right\} \\
& =\left\{\sum_{j=1}^{n} b_{j}\left(\left.\sum_{k=1}^{m} \psi_{k j}^{(2)}(0,0) \frac{\partial}{\partial x_{k}}\right|_{0}\right): b \in \Gamma\right\} \\
& =\left\{\left.\sum_{k=1}^{m}\left(\sum_{j=1}^{n} b_{j} \psi_{k j}^{(2)}(0,0)\right) \frac{\partial}{\partial x_{k}}\right|_{0}: b \in \Gamma\right\} \subset T_{0} X .
\end{aligned}
$$


Hence,

$$
\begin{aligned}
\left(\Gamma_{0}^{T}(\mathcal{W})\right)^{0} & =\left\{\xi \in T_{0}^{*} X \backslash\{0\} \simeq \mathbb{R}^{m} \backslash\{0\}: \xi \cdot v \geq 0 \text { for all } v \in \Gamma_{0}^{T}(\mathcal{W})\right\} \\
& =\left\{\xi \in \mathbb{R}^{m} \backslash\{0\}: \xi \cdot \Im A(0,0) b \geq 0 \text { for all } b \in \Gamma\right\}
\end{aligned}
$$

Therefore, since $\left(\Gamma_{0}^{T}(\mathcal{W})\right)^{0}$ is closed in $\mathbb{R}^{m} \backslash\{0\}$, we obtain

$$
\xi^{0} \notin\left(\Gamma_{0}^{T}(\mathcal{W})\right)^{0} \Leftrightarrow \exists \text { an open convex cone } \widetilde{\Gamma} \subset \subset \Gamma: \xi^{0} \cdot \Im A(0,0) \widetilde{\Gamma}<0 .
$$

For $j=1, \ldots, n$, define the vector fields

$$
L_{j}^{\prime}=L_{j}-\sum_{k=1}^{m} L_{j} Z_{k}(x, t) M_{k},
$$

where $M_{1}, \ldots, M_{m}$ are $C^{\infty}$ complex vector fields involving differentiation in the $x$ variables only such that

$$
M_{k} Z_{l}=\delta_{k l} \text { for all } 1 \leq k \leq m, 1 \leq l \leq m .
$$

Note that

$$
L_{j}^{\prime} Z_{l}=0 \text { for all } 1 \leq j \leq n, 1 \leq l \leq m .
$$

If $g(x, t)$ is any $C^{1}$ function defined in $\Omega$, observe that the differential

$$
d g(x, t)=\sum_{j=1}^{n} L_{j}^{\prime} g(x, t) d t_{j}+\sum_{k=1}^{m} M_{k} g(x, t) d Z_{k} .
$$

Hence, if we consider the $m$-form

$$
\omega(x, t)=g(x, t) d Z(x, t)=g(x, t) d Z_{1} \wedge \cdots \wedge d Z_{m}(x, t),
$$

its differential becomes

$$
d \omega(x, t)=\sum_{j=1}^{n} L_{j}^{\prime} g(x, t) d t_{j} \wedge d Z(x, t) .
$$

Since $f(x, t)$ is an approximate solution of $\mathcal{V}$ in $\mathcal{W}$,

$$
\forall N \in \mathbb{N} \exists C_{N}>0:\left|L_{j} f(x, t)\right| \leq C_{N}|t|^{N} \quad \text { for all }(x, t) \in \mathcal{W} .
$$

We also know that

$$
\lim _{\Gamma \ni t \rightarrow 0} \int_{X} f(x, t) \varphi(x) d x=\langle u, \varphi\rangle \text { exists for all } \varphi \in C_{0}^{\infty}(X) .
$$

Let $\eta(x) \in C_{0}^{\infty}\left(\mathbb{R}^{m}\right), \eta(x) \equiv 1$ for $|x| \leq r$, and $\eta(x) \equiv 0$ when $|x| \geq 2 r$ ( $r$ small). We will consider the following FBI transform of $\eta f$ :

$$
\mathcal{F}_{\eta f}(t ; y, \xi)=\int_{X} e^{i \xi \cdot(y-Z(x, t))-|\xi|\langle y-Z(x, t)\rangle^{2}} \eta(x) f(x, t)\left(\operatorname{det} Z_{x}(x, t)\right) d x .
$$


where for $z \in \mathbb{C}^{m}$, we write $\langle z\rangle^{2}=z_{1}^{2}+\cdots+z_{m}^{2}$. Since the boundary value $b f=u$ exists, we have

$$
\begin{aligned}
\mathcal{F}_{\eta f}(0 ; y, \xi) & =\int_{X} e^{i \xi \cdot(y-x)-|\xi|\langle y-x\rangle^{2}} \eta(x) u(x) d x \\
& =\mathcal{F}_{\eta u}(y, \xi)
\end{aligned}
$$

Let $\xi^{0} \in \mathbb{R}^{m} \backslash\{0\}$ be such that $\xi^{0} \notin\left(\Gamma_{0}^{T}(\mathcal{W})\right)^{0}$. Then, by (3.5), we can get an open convex cone $\widetilde{\Gamma} \subset \subset \Gamma$ such that

$$
\xi^{0} \cdot \Im A(0,0) \widetilde{\Gamma}<0
$$

Fix $T \in \widetilde{\Gamma}$ and let

$$
\gamma(s)=s T \text { for } 0 \leq s \leq 1 .
$$

Consider the $m$-form $\omega(x, t)=g(x, t) d Z(x, t)$, where

$$
g(x, t)=e^{i \xi \cdot(y-Z(x, t))-|\xi|\langle y-Z(x, t)\rangle^{2}} \eta(x) f(x, t),
$$

and it is to be understood that $y$ and $\xi$ are parameters. We now avail ourselves of Stokes' theorem

$$
\int_{\gamma} \int_{X} d \omega(x, t)=\int_{\partial(X \times \gamma)} \omega(x, t) .
$$

Using (3.7), equation (3.9) becomes

$$
\int_{\gamma} \int_{X} \sum_{j=1}^{n} L_{j}^{\prime} g(x, t) d t_{j} \wedge d Z(x, t)=\int_{X} \omega(x, T)-\int_{X} \omega(x, 0) .
$$

Note that by (3.6),

$$
\begin{aligned}
L_{j}^{\prime} g(x, t)= & e^{i \xi \cdot(y-Z(x, t))-|\xi|\langle y-Z(x, t)\rangle^{2}} \eta(x) L_{j}^{\prime} f(x, t) \\
& +e^{i \xi \cdot(y-Z(x, t))-|\xi|\langle y-Z(x, t)\rangle^{2}} f(x, t) L_{j}^{\prime} \eta(x, t), \\
\omega(x, T)= & g(x, T)\left(\operatorname{det} Z_{x}(x, T)\right) d x \\
= & e^{i \xi \cdot(y-Z(x, T))-|\xi|\langle y-Z(x, T)\rangle^{2}} \eta(x) f(x, T)\left(\operatorname{det} Z_{x}(x, T)\right) d x, \text { and } \\
\omega(x, 0)= & g(x, 0) d x=e^{i \xi \cdot(y-x)-|\xi|\langle y-x\rangle^{2}} \eta(x) u(x) d x .
\end{aligned}
$$

Hence, together with (3.10), the above equations imply

$$
\begin{aligned}
\left|\mathcal{F}_{\eta u}(y, \xi)\right| \leq & \left|\int_{X} e^{Q(x, T, y, \xi)} \eta(x) f(x, T)\left(\operatorname{det} Z_{x}(x, T)\right) d x\right| \\
& +\sum_{j=1}^{n}\left|\int_{\gamma} \int_{X} e^{Q(x, t, y, \xi)} \eta(x) L_{j}^{\prime} f(x, t)\left(\operatorname{det} Z_{x}(x, t)\right) d x d t_{j}\right| \\
& +\sum_{j=1}^{n}\left|\int_{\gamma} \int_{X} e^{Q(x, t, y, \xi)} f(x, t) L_{j}^{\prime} \eta(x) \operatorname{det} Z_{x} d x d t_{j}\right|
\end{aligned}
$$


where

$$
Q(x, t, y, \xi)=i \xi \cdot(y-Z(x, t))-|\xi|\langle y-Z(x, t)\rangle^{2} .
$$

We have

$$
\begin{aligned}
\Re Q(x, t, y, \xi)= & \xi \cdot \Im A(x, t) t-|\xi|\left[|y-x|^{2}+|\Re A(x, t) t|^{2}-|\Im A(x, t) t|^{2}\right. \\
& -2\langle y-x, \Re A(x, t) t\rangle] .
\end{aligned}
$$

Let $M>0$ such that

$$
\|A(x, t)-A(0,0)\| \leq M(|x|+|t|) \text { for all }(x, t) \in \Omega
$$

and so, for all $(x, t) \in \Omega$ :

$$
\xi \cdot \Im A(x, t) t \leq \xi \cdot \Im A(0,0) t+M|\xi||t|(|x|+|t|) .
$$

Therefore, for some $C>0$,

$$
\begin{aligned}
\Re Q(x, t, y, \xi) \leq & \xi \cdot \Im A(0,0) t+M(|x|+|t|)|t||\xi| \\
& +C|t|^{2}|\xi|-\frac{|y-x|^{2}}{2}|\xi| .
\end{aligned}
$$

Since $\xi^{0} \cdot(\Im A(0,0) T)<0$, there is a conic neighborhood $\mathcal{C}$ of $\xi^{0}$ and $c>0$ such that

$$
\xi \cdot(\Im A(0,0) t) \leq-2 c|t||\xi| \quad \forall \xi \in \mathcal{C}, \forall t \in \gamma .
$$

Hence for $r$ small enough, $|x| \leq r$, and $|t|$ small,

$$
\Re Q(x, t, y, \xi) \leq-c|t||\xi| \quad \forall \xi \in \mathcal{C}, \forall t \in \gamma .
$$

Thus, there are $\delta>0, C_{0}>0$, an open neighborhood $\mathcal{O} \subset \mathbb{R}^{m}$ of the origin and an open conic neighborhood $\mathcal{C} \subset \mathbb{R}^{m} \backslash\{0\}$ of $\xi^{0}$ such that for all $t \in \gamma$ and all $(y, \xi) \in$ $\mathcal{O} \times \mathcal{C}:$

$$
\Re Q(x, t, y, \xi) \leq-\frac{1}{4} C_{0}|t||\xi| .
$$

We are now ready to conclude the proof. We consider each term in (3.11). Since $f(., T)$ is a distribution, and

$$
\Re Q(x, T, y, \xi) \leq-\frac{1}{4} C_{0}|T||\xi|,
$$

for some $C, C_{0}^{\prime}>0$, we have:

$$
\begin{aligned}
& \left|\int_{X} e^{i \xi \cdot(y-Z(x, T))-|\xi|\langle y-Z(x, T)\rangle^{2}} \eta(x) f(x, T)\left(\operatorname{det} Z_{x}(x, T)\right) d x\right| \\
\leq & C e^{-\frac{1}{4} C_{0}^{\prime}|\xi|} \text { for all }(y, \xi) \in \mathcal{O} \times \mathcal{C} .
\end{aligned}
$$

Since $L_{j}^{\prime} \eta(x) \equiv 0$ for $|x| \leq r$, the term

$$
\left|\int_{\gamma} \int_{X} e^{i \xi \cdot(y-Z(x, t))-|\xi|\langle y-Z(x, t)\rangle^{2}} L_{j}^{\prime} \eta(x) f(x, t)\left(\operatorname{det} Z_{x}(x, t)\right) d x d t_{j}\right|
$$


has an exponential decay for $y$ near 0 and $\xi$ in a conic neighborhood of $\xi_{0}$. To estimate the third term, for $N$ a positive integer,

$$
\begin{aligned}
& |\xi|^{N} \int_{\gamma}\left|\int_{X} e^{i \xi \cdot(y-Z(x, t))-|\xi|\langle y-Z(x, t)\rangle^{2}} \eta(x) L_{j}^{\prime} f(x, t) d x\right| d t_{j} \\
\leq & C|\xi|^{N} \int_{\gamma}\left|\int_{X} e^{i \xi \cdot(y-Z(x, t))-|\xi|\langle y-Z(x, t)\rangle^{2}} \eta(x) L_{j} f(x, t) d x\right| d t_{j} \\
& +C|\xi|^{N} \sum_{k=1}^{m} \int_{\gamma}\left|\int_{X} e^{i \xi \cdot(y-Z(x, t))-|\xi|\langle y-Z(x, t)\rangle^{2}} \eta(x) L_{j} Z_{k}(x, t) M_{k} f(x, t) d x\right| d t_{j} .
\end{aligned}
$$

Since $f$ is an approximate solution of the $L_{j}$ 's, we obtain

$$
\begin{aligned}
& C|\xi|^{N} \int_{\gamma}\left|\int_{X} e^{i \xi \cdot(y-Z(x, t))-|\xi|\langle y-Z(x, t)\rangle^{2}} \eta(x) L_{j} f(x, t) d x\right| d t_{j} \\
\leq & C C_{N} \int_{\gamma} \int_{X} e^{-\frac{1}{4} C_{0}|t||\xi|}|\xi|^{N}|t|^{N} d x d t_{j} \\
\leq & C^{\prime} \text { for all }(y, \xi) \in \mathcal{O} \times \mathcal{C} .
\end{aligned}
$$

Since $b f=u$ exists, so does $b\left(M_{k} f\right)$ for all $k=1, \ldots, m$. Hence, after decreasing $\delta$, we can find a positive integer $J$ independent of $N$ such that

$$
\begin{aligned}
& C|\xi|^{N} \sum_{k=1}^{m} \int_{\gamma}\left|\int_{X} e^{i \xi \cdot(y-Z(x, t))-|\xi|\langle y-Z(x, t)\rangle^{2}} \eta(x) L_{j} Z_{k}(x, t) M_{k} f(x, t) d x\right| d t_{j} \\
\leq & K_{1}|\xi|^{N} \sum_{k=1}^{m} \int_{\gamma} \sum_{|\alpha| \leq J} \sup \left|D_{x}^{\alpha}\left\{e^{i \xi \cdot(y-Z(x, t))-|\xi|\langle y-Z(x, t)\rangle^{2}} \eta(x) L_{j} Z_{k}(x, t)\right\}\right| d t_{j} \\
\leq & K_{2} e^{-\frac{1}{4} C_{0}|t||\xi|}|\xi|^{N}|t|^{N} \\
\leq & C^{\prime \prime} \text { for all }(y, \xi) \in \mathcal{O} \times \mathcal{C} .
\end{aligned}
$$

Therefore, for each $N \in \mathbb{N}$ there exists a constant $C_{N}>0$ such that for all $(y, \xi) \in$ $O \times \mathcal{C}:$

$$
\left|\mathcal{F}_{\eta u}(y, \xi)\right| \leq \frac{C_{N}}{|\xi|^{N}}
$$

This shows that the FBI transform of $u, \mathcal{F}_{\eta u}(x, \xi)$, has rapid decay in $\xi$ for all $(x, \xi) \in$ $O \times \mathcal{C}$. It is well known (see [BH1] for example) that this implies

$$
\left(0, \xi^{0}\right) \notin W F(u) .
$$

This concludes the proof.

Corollary 3.1. (Edge-of-the-Wedge Theorem) Let $\mathcal{W}^{+}$and $\mathcal{W}^{-}$be wedges in $\Omega$ with edge $X$ whose directions are opposite: $\Gamma_{p}\left(\mathcal{W}^{+}\right)=-\Gamma_{p}\left(\mathcal{W}^{-}\right)$. If $u \in \mathcal{D}^{\prime}(X)$ is the boundary value of an approximate solution $f^{+}$of $\mathcal{V}$ on $\mathcal{W}^{+}$and also the boundary value of an approximate solution $f^{-}$of $\mathcal{V}$ on $\mathcal{W}^{-}$, then $W F_{p}(u) \subset i_{X}^{*}\left(T_{p}^{0}\right)$.

Proof. By Theorem 3.1,

$$
W F_{p}(u) \subset\left(\Gamma_{p}^{T}\left(\mathcal{W}^{+}\right)\right)^{0} \cap\left(\Gamma_{p}^{T}\left(\mathcal{W}^{-}\right)\right)^{0} .
$$


Note that

$$
\Gamma_{p}^{T}\left(\mathcal{W}^{+}\right)=-\Gamma_{p}^{T}\left(\mathcal{W}^{-}\right)
$$

Thus, if $\xi^{0} \in W F_{p}(u)$, then

$$
\xi^{0} \cdot \Gamma_{p}^{T}\left(\mathcal{W}^{+}\right) \geq 0 \text { and } \xi^{0} \cdot \Gamma_{p}^{T}\left(\mathcal{W}^{-}\right) \geq 0
$$

This implies that

$$
\xi^{0} \cdot \Gamma_{p}^{T}\left(\mathcal{W}^{+}\right)=0
$$

Since $\Gamma_{p}^{T}\left(\mathcal{W}^{+}\right)$is open in $\Re \mathcal{V}_{p} \cap T_{p} X$, we conclude that

$$
\xi^{0} \in\left(\Re \mathcal{V}_{p} \cap T_{p} X\right)^{\perp}=i_{X}^{*}\left(T_{p}^{0}\right) .
$$

Thus, $W F_{p}(u) \subset i_{X}^{*}\left(T_{p}^{0} \Omega\right)$.

COROLlaRY 3.2. If $(M, \mathcal{V})$ is an elliptic structure and we have the same hypothesis as in the previous corollary, then $u$ is $C^{\infty}$ in $X$.

We will next prove the converse of Theorem 3.1.

TheOREM 3.2. Let $(M, \mathcal{V})$ be an involutive structure, $\operatorname{dim}_{\mathbb{R}} M=m+n$, rank of $\mathcal{V}=n, X \subset M$ a maximally real submanifold, and $\mathcal{W}$ a wedge in $M$ with edge $X$. Suppose $u \in \mathcal{E}^{\prime}(X)$ is such that

$$
W F(u) \subset\left(\Gamma^{T}(\mathcal{W})\right)^{0} .
$$

Then in a slightly smaller wedge $\mathcal{W}^{\prime} \subset \subset \mathcal{W}$ with edge $X$, there exists an approximate solution $f \in \mathcal{D}^{\prime}\left(\mathcal{W}^{\prime}\right)$ such that

$$
u=b f \quad \text { on } X \text {. }
$$

Proof. We take off from (3.4). For some open convex cone $\Gamma^{\prime} \subset \subset \Gamma$, one can write

$$
\mathcal{W}^{\prime}=B_{r}(0) \times \Gamma^{\prime} .
$$

If $\Gamma^{T}(\mathcal{W})^{0}=\{0\}$, then $u$ is $C^{\infty}$, in which case the conclusion of Theorem 3.2 is well known. We may therefore assume that $\Gamma^{T}(\mathcal{W})^{0} \neq\{0\}$. Using (3.4) and the fact that $\Gamma^{\prime} \subset \subset \Gamma$, one can find an open convex cone $\mathcal{C} \subset \mathbb{R}^{m} \backslash\{0\}$ containing $\left(\Gamma_{0}^{T}(\mathcal{W})\right)^{0}$ and a constant $c>0$ such that

$$
\xi \cdot \Im A(0,0) t \geq c|\xi||t| \quad \text { for all }(\xi, t) \in \mathcal{C} \times \Gamma^{\prime} .
$$

For $(x, t) \in \mathcal{W}^{\prime}$ and $\xi \in \mathcal{C}$, define

$$
\begin{aligned}
Q(x, t, \xi) & =i \xi \cdot Z(x, t) \\
& =i \xi \cdot(x+\Re A(x, t) t)-\xi \cdot \Im A(x, t) t .
\end{aligned}
$$

From (3.13) and the fact that $\Im A(x, t)$ is of class $C^{1}$ near $(0,0)$, one obtains for some $M>0$ and for all $(x, t) \in \mathcal{W}^{\prime}$ and $\xi \in \mathcal{C}$ :

$$
\begin{aligned}
\Re Q(x, t, \xi) & =-\xi \cdot \Im A(x, t) t \\
& \leq-\xi \cdot \Im A(0,0) t+M|\xi||t|(|x|+|t|) \\
& \leq-c|\xi||t|+M|\xi||t|(|x|+|t|)
\end{aligned}
$$


Choosing $0<r, \delta<\frac{c}{4 M}$, we can insure that

$$
\Re Q(x, t, \xi) \leq-\frac{c}{2}|\xi||t| \quad \text { for all }(x, t, \xi) \in B_{r}(0) \times \Gamma_{\delta}^{\prime} \times \mathcal{C} .
$$

Since $u \in \mathcal{E}^{\prime}(X)$, there exists a constant $C>0$ and a positive integer $N$ such that the Fourier transform

$$
|\widehat{u}(\xi)| \leq C(1+|\xi|)^{N} \quad \text { for all } \xi \in \mathbb{R}^{m} .
$$

This allows us to define for $(x, t) \in B_{r}(0) \times \Gamma_{\delta}^{\prime}$ the continuous function

$$
\begin{aligned}
f_{1}(x, t) & =\frac{1}{(2 \pi)^{m}} \int_{\mathcal{C}} e^{Q(x, t, \xi)} \widehat{u}(\xi) d \xi \\
& =\frac{1}{(2 \pi)^{m}} \int_{\mathcal{C}} e^{i \xi \cdot Z(x, t)} \widehat{u}(\xi) d \xi .
\end{aligned}
$$

We claim that (i) $f_{1}$ is an approximate solution of $\mathcal{V}$ and for some $C>0$, (ii) $\left|f_{1}(x, t)\right||t|^{N} \leq C$ where $N$ is the same as the one in (3.15). Assuming that the claims are true for the moment, we can use Theorem 4.1 from Section 4 to guarantee the existence of the boundary value $b f_{1}=\lim _{\Gamma_{\delta}^{\prime} \ni t \rightarrow 0} f_{1}(., t)$ in $\mathcal{D}^{\prime}\left(B_{r}(0)\right)$ and moreover,

$$
b f_{1}(x)=\frac{1}{(2 \pi)^{m}} \int_{\mathcal{C}} e^{i \xi \cdot x} \widehat{u}(\xi) d \xi .
$$

To show (i), we fix $t_{0} \in \Gamma_{\delta}^{\prime}$ and we consider a small open neighborhood of $t_{0}$ in $\Gamma_{\delta}^{\prime}$. In this small neighborhood, estimate (3.14) allows us to pass $L_{j}$ under the integral sign

$$
L_{j} f_{1}(x, t)=\frac{1}{(2 \pi)^{m}} \int_{\mathcal{C}} i\left(\xi \cdot L_{j} Z(x, t)\right) e^{i \xi \cdot Z(x, t)} \widehat{u}(\xi) d \xi .
$$

Since the $Z_{k}(x, t)$ are approximate first integrals for $\mathcal{V}$, for each $l=1,2, \ldots$ there exists a constant $C_{l}>0$ such that

$$
\left|L_{j} Z_{k}(x, t)\right| \leq C_{l}|t|^{l} \quad \text { for all }(x, t) \in B_{r}(0) \times B_{\delta}(0), \forall k .
$$

From (3.14) and (3.17), for each $l=1,2, \ldots$, there exists a constant $K_{l}>0$ such that

$$
\left|L_{j} f_{1}(x, t)\right| \leq K_{l}|t|^{l} \quad \text { for all }(x, t) \in B_{r}(0) \times \Gamma_{\delta}^{\prime} .
$$

Hence, $f_{1}$ is an approximate solution of $\mathcal{V}$ and claim (i) is proved. Claim (ii) follows from (3.14) and (3.15)which imply that there is a constant $C^{\prime}>0$ such that

$$
\left|f_{1}(x, t)\right||t|^{N} \leq C^{\prime} \quad \text { for all }(x, t) \in B_{r}(0) \times \Gamma_{\delta}^{\prime} .
$$

For $x \in B_{r}(0)$ define

$$
v(x)=\frac{1}{(2 \pi)^{m}} \int_{\mathbb{R}^{m} \backslash \mathcal{C}} e^{i \xi \cdot x} \widehat{u}(\xi) d \xi .
$$

Using the fact that $W F_{0}(u) \subset\left(\Gamma_{0}^{T}(\mathcal{W})\right)^{0}$, compactness of $\left(\mathbb{R}^{m} \backslash \mathcal{C}\right) \cap \mathbb{S}^{m-1}$, and the characterization of the $C^{\infty}$ wavefront set by the rapid decay of the Fourier transform, we get that $v \in C^{\infty}\left(B_{r}(0)\right)$. It is well known (see [A] for example) that in this case, one can find a $C^{\infty}$ function $f_{2} \in C^{\infty}\left(B_{r}(0) \times B_{\delta}(0)\right)$ such that $f_{2}$ is an approximate solution of $\mathcal{V}$ and $b f_{2}=v$ on $X$. Thus, from (3.16) and (3.18) we get

$$
u=b f_{1}+b f_{2}=b f
$$

where $f=f_{1}+f_{2}$ is an approximate solution of $\mathcal{V}$ in the wedge $\mathcal{W}^{\prime}$. This completes the proof. $\mathrm{Q}$ 
4. Existence of Boundary Values. In this section we present a sufficient condition for a function defined on a wedge to have a boundary value on the edge of the wedge. The result is more general than what was needed in the proof of Theorem 3.2 .

We will consider a system of smooth complex vector fields

$$
L_{j}=\frac{\partial}{\partial t_{j}}+\sum_{k=1}^{m} a_{j k}(x, t) \frac{\partial}{\partial x_{k}}
$$

in a neighborhood $U$ of the origin in $\mathbb{R}_{x}^{m} \times \mathbb{R}_{t}^{n}$. For simplicity, say $U=B_{r}(0) \times B_{\delta}(0)$ and let $\mathcal{W}=B_{r}(0) \times \Gamma_{\delta}$ be a wedge where $\Gamma_{\delta} \subset \mathbb{R}_{t}^{n}$ is a truncated open convex cone. For analogues of the following theorem for a single vector field see Theorem 1.1 in [BH2] and Theorem VI.1.3 in $[\mathrm{BCH}]$ :

THEOREM 4.1. Let $\mathcal{W}=B_{r}(0) \times \Gamma_{\delta}$ be as above and suppose that $f(x, t) \in C(\mathcal{W})$ satisfies: for some $C>0$ and some $N \in \mathbb{N}$

(i)

$$
\int_{B_{r}(0)}\left|L_{j} f(x, t)\right| d x \leq C
$$

and (ii)

$$
|f(x, t)||t|^{N} \leq C
$$

Then $b f=\lim _{\Gamma_{\delta} \ni t \rightarrow 0} f(., t)$ exists in $\mathcal{D}^{\prime}\left(B_{r}(0)\right)$.

Proof. Let $Z_{1}, \ldots, Z_{m}: U \rightarrow \mathbb{C}$ be smooth functions near the origin in $U$ satisfying

$$
L_{j} Z_{k}(x, t)=O\left(|t|^{l}\right) \text { for } l=1,2, \ldots, \text { and } Z_{k}(x, 0)=x_{k}, \quad 1 \leq k \leq m .
$$

Define

$$
b_{j k}(x, t)=L_{j} Z_{k}(x, t)
$$

Write

$$
Z(x, t)=\left(Z_{1}(x, t), \ldots, Z_{m}(x, t)\right) \quad \text { and } \quad Z_{k}(x, t)=\Psi_{1 k}(x, t)+i \Psi_{2 k}(x, t),
$$

where $\Psi_{1 k}(x, t)$ and $\Psi_{2 k}(x, t)$ are real-valued. For $j=1, \ldots, m$, let

$$
M_{j}=\sum_{k=1}^{m} c_{j k}(x, t) \frac{\partial}{\partial x_{k}}
$$

be vector fields in $x$-space satisfying

$$
M_{j} Z_{k}=\delta_{j k}, \quad\left[M_{j}, M_{k}\right]=0 .
$$

Note that for each $j, k$,

$$
\left[M_{j}, L_{k}\right]=\sum_{l=1}^{m} d_{j k l}(x, t) M_{l}
$$


where each $d_{j k l}(x, t)=O\left(|t|^{s}\right)$ for $s=1,2, \ldots$ Indeed, the latter can be seen by expressing $\left[M_{j}, L_{k}\right]$ in terms of the basis $\left\{L_{1}, \ldots, L_{n}, M_{1}, \ldots, M_{m}\right\}$ and applying both sides to the $n+m$ functions $\left\{t_{1}, \ldots, t_{n}, Z_{1}, \ldots, Z_{m}\right\}$. Equations (4.1) and (4.2) imply that

$$
M_{k} b_{j k}=O\left(|t|^{s}\right) \text { for } s=1,2, \ldots
$$

If $g(x, t)$ is any $C^{1}$ function defined in $U$, observe that the differential

$$
d g=\sum_{k=1}^{m} M_{k}(g) d Z_{k}+\sum_{j=1}^{n} L_{j}(g) d t_{j}-\sum_{j=1}^{n} \sum_{k=1}^{m} M_{k}(g) b_{j k} d t_{j} .
$$

Hence, if we consider the $m$-form $\omega=g d Z$, we get

$$
d \omega=d g \wedge d Z=\sum_{j=1}^{n} L_{j}(g) d t_{j} \wedge d Z-\sum_{j=1}^{n} \sum_{k=1}^{m} M_{k}(g) b_{j k} d t_{j} \wedge d Z .
$$

Observe that hypothesis (ii) in the theorem together with the fact that

$$
b_{j k}(x, t)=O\left(|t|^{s}\right), \quad M_{k} b_{j k}(x, t)=O\left(|t|^{s}\right) \quad \forall s
$$

imply that $\forall \varphi \in C_{0}^{\infty}\left(B_{r}(0)\right)$,

$$
\left|\int_{\Gamma_{\delta}} \int_{B_{r}(0)} b_{j k}(x, t) M_{k} f(x, t) \varphi(x) d x d t\right| \leq C_{2},
$$

where $C_{2}>0$ is a constant that depends only on $\sup _{x \in B_{r}(0)} \sum_{|\alpha| \leq 1}\left\|D^{\alpha} \varphi(x)\right\|$. Let

$$
\Psi_{1}=\left(\Psi_{11}, \ldots, \Psi_{1 m}\right) \text { and } \Psi_{2}=\left(\Psi_{12}, \ldots, \Psi_{2 m}\right) .
$$

For $\varphi \in C_{0}^{\infty}\left(B_{r}(0)\right)$ and $k$ a nonnegative integer, define

$$
T_{k} \varphi(x, t)=\sum_{|\alpha| \leq k} \frac{i^{|\alpha|}}{\alpha !}\left[\left(\frac{\partial}{\partial x}\right)^{\alpha} \varphi\left(\Psi_{1}(x, t)\right)\right]\left(\Psi_{2}(x, t)\right)^{\alpha} .
$$

We will first show that $\lim _{\Gamma_{\delta} \ni t \rightarrow 0} \int_{B_{r}(0)} f(x, t)\left(T_{N} \varphi\right)(x, t) d Z(x, t)$ exists. To prove this, fix $T \in \Gamma_{\delta}$ and let $\delta^{\prime}=\delta-|T|$. For $s \in \Gamma_{\delta^{\prime}}$, define $\gamma_{s}(\tau)=(1-\tau) s+\tau T, 0 \leq \tau \leq 1$. Let $\omega=\left(f T_{N} \varphi\right) d Z$. Using (4.3) and Stokes' theorem, we get

$$
\begin{aligned}
& \int_{B_{r}(0)} f(x, s)\left(T_{N} \varphi\right)(x, s) d Z(x, s)=\int_{B_{r}(0)} f(x, T)\left(T_{N} \varphi\right)(x, T) d Z(x, T) \\
& -\sum_{j=1}^{n} \int_{B_{r}(0)} \int_{\gamma_{s}}\left(L_{j} f-\sum_{k=1}^{m} M_{k}(f) b_{j k}\right) T_{N} \varphi d t_{j} \wedge d Z \\
& -\sum_{j=1}^{n} \int_{B_{r}(0)} \int_{\gamma_{s}}\left(L_{j} T_{N} \varphi-\sum_{k=1}^{m} M_{k}\left(T_{N} \varphi\right) b_{j k}\right) f d t_{j} \wedge d Z .
\end{aligned}
$$


The second integral on the RHS has a limit as $s \rightarrow 0$ by hypothesis $(i)$ of the theorem and an argument similar to the one used to get (4.4). For the third integral, consider

$$
\begin{aligned}
L_{j} T_{N} \varphi= & \sum_{|\alpha| \leq N} \frac{i^{|\alpha|}}{\alpha !}\left[L_{j}\left(\frac{\partial}{\partial x}\right)^{\alpha} \varphi\left(\Psi_{1}\right)\right]\left(\Psi_{2}\right)^{\alpha} \\
& +\sum_{|\alpha| \leq N} \frac{i^{|\alpha|}}{\alpha !}\left[\left(\frac{\partial}{\partial x}\right)^{\alpha} \varphi\left(\Psi_{1}\right)\right] L_{j}\left(\Psi_{2}\right)^{\alpha} \\
= & \sum_{|\alpha| \leq N} \sum_{l=1}^{m} \frac{i^{|\alpha|}}{\alpha !}\left(\left(\frac{\partial}{\partial x}\right)^{\alpha+e_{l}} \varphi\left(\Psi_{1}\right)\right)\left(L_{j} \Psi_{1 l}\right)\left(\Psi_{2}\right)^{\alpha} \\
& +\sum_{1 \leq|\alpha| \leq N} \sum_{l=1}^{m} \frac{i^{|\alpha|}}{\alpha !}\left(\frac{\partial}{\partial x}\right)^{\alpha} \varphi\left(\Psi_{1}\right)\left[\alpha_{l}\left(\Psi_{2}\right)^{\alpha-e_{l}} L_{j} \Psi_{2 l}\right] \\
= & \sum_{|\alpha| \leq N} \sum_{l=1}^{m} \frac{i^{|\alpha|}}{\alpha !}\left(\left(\frac{\partial}{\partial x}\right)^{\alpha+e_{l}} \varphi\left(\Psi_{1}\right)\right)\left(L_{j} \Psi_{1 l}\right)\left(\Psi_{2}\right)^{\alpha} \\
& +\sum_{|\alpha| \leq N-1} \sum_{l=1}^{m} \frac{i^{|\alpha|+1}}{\alpha !}\left(\frac{\partial}{\partial x}\right)^{\alpha+e_{l}} \varphi\left(\Psi_{1}\right)\left(L_{j} \Psi_{2 l}\right)\left(\Psi_{2}\right)^{\alpha} \\
= & \sum_{|\alpha|=N} \sum_{l=1}^{m} \frac{i^{|\alpha|}}{\alpha !}\left(\left(\frac{\partial}{\partial x}\right)^{\alpha+e_{l}} \varphi\left(\Psi_{1}\right)\right)\left(L_{j} \Psi_{1 l}\right)\left(\Psi_{2}\right)^{\alpha} \\
+ & \sum_{|\alpha| \leq N-1} \sum_{l=1}^{m} \frac{i^{|\alpha|}}{\alpha !}\left(\frac{\partial}{\partial x}\right)^{\alpha+e_{l}} \varphi\left(\Psi_{1}\right)\left(\Psi_{2}\right)^{\alpha} L_{j}\left(Z_{l}\right) .
\end{aligned}
$$

Since $Z(x, 0)=x,\left|\Psi_{2}(x, t)\right|=\left|\Psi_{2}(x, t)-\Psi_{2}(x, 0)\right| \leq C^{\prime}|t|$ and so, recalling that the $Z_{l}$ are approximate solutions, we conclude that

$$
\left|L_{j} T_{N} \varphi(x, t)\right| \leq C_{j}^{\prime}|t|^{N} .
$$

Hence,

$$
\lim _{\Gamma_{\delta} \ni t \rightarrow 0} \int_{B_{r}(0)} f(x, t) T_{N} \varphi(x, t) d Z(x, t) \text { exists. }
$$

We will next use the existence of

$$
\lim _{\Gamma_{\delta} \ni t \rightarrow 0} \int_{B_{r}(0)} f(x, t)\left(T_{N} g\right)(x, t) d Z(x, t)
$$

to show that

$$
\lim _{\Gamma_{\delta} \ni t \rightarrow 0} \int_{B_{r}(0)} f(x, t)\left(T_{N-1} g\right)(x, t) d Z(x, t) \text { exists. }
$$

To do so, let $\psi(x, t) \in C_{0}^{\infty}\left(B_{r}(0) \times B_{\delta}(0)\right)$ and for a fixed multi-index $\beta$ with $|\beta|=N$ let

$$
g(x, t)=\tilde{\psi}(x, t) \widetilde{\Psi}_{2}(x, t)^{\beta},
$$


where $\tilde{\psi}(x, t)=\psi\left(\Psi_{1}(x, t), t\right)$ and $\widetilde{\Psi}_{2}(x, t)=\Psi_{2}\left(\Psi_{1}(x, t), t\right)$. The functions $\tilde{\psi}$ and $\widetilde{\Psi}_{2}(x, t)$ exist since the map $(x, t) \rightarrow\left(\Psi_{1}(x, t), t\right)$ is a diffeomorphism. Note that we may write

$$
\begin{aligned}
T_{N}\left(\tilde{\psi} \widetilde{\Psi}_{2}^{\beta}\right)(x, t) & =\psi(x, t) \Psi_{2}(x, t)^{\beta}+\psi(x, t) \sum_{|\alpha|=N} a_{\alpha}(x, t) \Psi_{2}(x, t)^{\alpha} \\
& +\sum_{|\gamma|>N} b_{\gamma}(x, t) \Psi_{2}(x, t)^{\gamma}
\end{aligned}
$$

( $t$ is treated as a parameter in $T_{N} g(x, t)$ and $T_{N}$ acts on $x \rightarrow g(x, t)$ ) where $a_{\alpha}(x, t)$ and $b_{\gamma}(x, t)$ are smooth and $a_{\alpha}(x, 0) \equiv 0$. The assumption on the growth of $f$ implies that the limit of

$$
\int_{B_{r}(0)} f(x, t)\left(\psi(x, t) \sum_{|\alpha|=N} a_{\alpha}(x, t) \Psi_{2}(x, t)^{\alpha}+\sum_{|\gamma|>N} b_{\gamma}(x, t) \Psi_{2}(x, t)^{\gamma}\right) d Z
$$

exists as $t \rightarrow 0, t \in \Gamma_{\delta}$. From (4.5), it follows that for any $\psi(x, t) \in C_{0}^{\infty}\left(B_{r}(0) \times B_{\delta}(0)\right)$ and any multi-index $\beta$ with $|\beta|=N$,

$$
\lim _{\Gamma_{\delta} \ni t \rightarrow 0} \int_{B_{r}(0)} f(x, t) \psi(x, t) \Psi_{2}(x, t)^{\beta} d Z(x, t)
$$

exists. Note next that for any $g(x, t) \in C_{0}^{\infty}\left(B_{r}(0) \times B_{\delta}(0)\right)$,

$$
T_{N} g(x, t)=T_{N-1} g(x, t)+\sum_{|\beta|=N} \psi_{\beta}(x, t) \Psi_{2}(x, t)^{\beta}
$$

for some smooth $\psi_{\beta}$ of compact support. Hence, from (4.5) and (4.6),

$$
\lim _{\Gamma_{\delta} \ni t \rightarrow 0} \int_{B_{r}(0)} f(x, t)\left(T_{N-1} g\right)(x, t) d Z(x, t) \quad \text { exists. }
$$

We will prove by descending induction that for any $g(x, t) \in C_{0}^{\infty}\left(B_{r}(0) \times B_{\delta}(0)\right)$ and $0 \leq k \leq N$,

$$
\lim _{t \rightarrow 0} \int_{B_{r}(0)} f(x, t) T_{k} g(x, t) d Z(x, t) \quad \text { exists, }
$$

which for $k=0$ and $g(x, t)=\psi(x) \in C_{0}^{\infty}\left(B_{r}(0)\right)$ proves the Theorem. To proceed by induction, suppose $1 \leq k \leq N$ and assume that for any multi-index $\beta$ with $|\beta|=k$, the limits

$$
\begin{aligned}
& \lim _{t \rightarrow 0} \int_{B_{r}(0)} f(x, t) \Psi_{2}(x, t)^{\beta} g(x, t) d Z(x, t) \text { and } \\
& \lim _{t \rightarrow 0} \int_{B_{r}(0)} f(x, t) T_{k-1} g(x, t) d Z(x, t)
\end{aligned}
$$

both exist for any $g(x, t) \in C_{0}^{\infty}\left(B_{r}(0) \times B_{0}(r)\right)$. We have already seen in (4.6) and (4.7) that (4.8) is true for $k=N$. Fix $\beta^{\prime}$ with $\left|\beta^{\prime}\right|=k-1$. Plug $g(x, t)=\tilde{\psi}(x, t) \widetilde{\Psi}_{2}(x, t)^{\beta^{\prime}}$ in the limit on the right in (4.8) and observe that $T_{k-1} g$ may be written as

$$
\begin{aligned}
T_{k-1} g(x, t) & =\psi(x, t) \Psi_{2}(x, t)^{\beta \prime}+\psi(x, t) \sum_{|\alpha|=k-1} c_{\alpha}(x, t) \Psi_{2}(x, t)^{\alpha} \\
& +\sum_{|\gamma| \geq k} d_{\gamma}(x, t) \Psi_{2}(x, t)^{\gamma}
\end{aligned}
$$


where $c_{\alpha}(x, t)$ and $d_{\gamma}(x, t)$ are smooth and $c_{\alpha}(x, 0) \equiv 0$. From the existence of the two limits in (4.8) we derive that

$$
\lim _{t \rightarrow 0} \int_{B_{r}(0)} f(x, t)\left(\psi(x, t) \Psi_{2}(x, t)^{\beta \prime}+\psi(x, t) \sum_{|\alpha|=k-1} c_{\alpha}(x, t) \Psi_{2}(x, t)^{\alpha}\right) d Z(x, t)
$$

exists. Observe next that since each $c_{\alpha}(x, 0) \equiv 0$, given any collection $\left\{\psi_{\beta}(x, t):|\beta|=\right.$ $k-1\}$ of compactly supported functions, we can find compactly supported functions $\left\{\eta_{\beta^{\prime}}(x, t):\left|\beta^{\prime}\right|=k-1\right\}$ such that

$$
\sum_{\beta^{\prime}} \eta_{\beta^{\prime}} \Psi_{2}^{\beta^{\prime}}+\sum_{\beta^{\prime}} \eta_{\beta^{\prime}}\left(\sum_{\alpha} c_{\alpha} \Psi_{2}^{\alpha}\right)=\sum_{\beta} \psi_{\beta} \Psi_{2}^{\beta} .
$$

We conclude that

$$
\lim _{t \rightarrow 0} \int_{B_{r}(0)} f(x, t) \Psi_{2}(x, t)^{\beta} \psi(x, t) d Z(x, t) \quad \text { exists }
$$

for all $\beta$ with $|\beta|=k-1$ and $\psi(x, t) \in C^{\infty}\left(B_{r}(0) \times B_{r}(0)\right)$. Hence, taking account of (4.8) and (4.9) we conclude that

$$
\lim _{t \rightarrow 0} \int_{B_{r}(0)} f(x, t) T_{k-2} g(x, t) d Z(x, t) \quad \text { exists. }
$$

We have thus proved that (4.8) holds for $k-1$, completing the inductive step. Therefore,

$$
\lim _{t \rightarrow 0} \int_{B_{r}(0)} f(x, t) \psi(x) d Z(x, t) \quad \text { exists }
$$

and thus $b f=\lim _{t \rightarrow 0} f(., t)$ exists. $\square$

For the rest of this section, let $(M, \mathcal{V})$ be $\mathbb{R}^{m+n}=\mathbb{R}_{x}^{m} \times \mathbb{R}_{t}^{n}$ with a CR structure $\mathcal{V}$ near the origin; i.e., $\mathcal{V} \cap \overline{\mathcal{V}}=\{0\}$ in a neighborhood $U=B_{r}(0) \times B_{\delta}(0)$ of the origin in $\mathbb{R}_{x}^{m} \times \mathbb{R}_{t}^{n}$. Suppose that $\mathcal{V}$ is generated in $U$ by the complex vector fields $\left\{L_{1}, \ldots, L_{n}\right\}$, where

$$
L_{j}=\frac{\partial}{\partial t_{j}}+\sum_{k=1}^{m} a_{j k}(x, t) \frac{\partial}{\partial x_{k}} .
$$

Let $Z_{1}, \ldots, Z_{m}: U \rightarrow \mathbb{C}$ be a complete set of smooth approximate first integrals for $\mathcal{V}$ in $U$ such that

$$
Z_{l}(x, 0)=x_{l}, \quad 1 \leq l \leq m .
$$

For each $l=1, \ldots, m$, we may write

$$
Z_{l}(x, t)=x_{l}+\sum_{s=1}^{n} t_{s} \psi_{l s}(x, t)
$$

where $\psi_{l s}(x, t)=\psi_{l s}^{(1)}(x, t)+i \psi_{l s}^{(2)}(x, t)$. Since $\mathcal{V}$ is CR in $U$, for each $1 \leq j \leq n$ there exists $1 \leq j^{\prime} \leq m$ such that

$$
\Im a_{j j^{\prime}}(0,0) \neq 0 .
$$


Observe that

$$
\Im a_{j l}(0,0)=-\psi_{l j}^{(2)}(0,0)
$$

Indeed,

$$
\begin{aligned}
L_{j} Z_{l}(x, t) & =\frac{\partial Z_{l}}{\partial t_{j}}(x, t)+\sum_{k=1}^{m} a_{j k}(x, t) \frac{\partial Z_{l}}{\partial x_{k}}(x, t) \\
& =\left(\sum_{s=1}^{n} t_{s} \frac{\partial \psi_{l s}}{\partial t_{j}}(x, t)+\psi_{l j}(x, t)\right) \\
& +\left(\sum_{k=1}^{m} a_{j k}(x, t)\left(\delta_{k l}+\sum_{s=1}^{n} t_{s} \frac{\partial \psi_{l s}}{\partial x_{k}}(x, t)\right)\right) .
\end{aligned}
$$

Evaluating this at $(0,0)$, we get

$$
0=\psi_{l j}(0,0)+a_{j l}(0,0)
$$

Corollary 4.1. Let $\mathcal{W}=B_{r}(0) \times \Gamma_{\delta}$ be a wedge with edge $B_{r}(0)$, where $\Gamma \subset \mathbb{R}_{t}^{n}$ is an open cone with vertex at the origin, and suppose that $f(x, t) \in C(\mathcal{W})$ satisfies: for some $C>0$ and some $N \in \mathbb{N}$,

$$
\text { (i) } \quad \int_{B_{r}(0)}\left|L_{j} f(x, t)\right| d x \leq C
$$

and

$$
\text { (ii) }|f(x, t)||Z(x, t)-Z(x, 0)|^{N} \leq C .
$$

Then $b f=\lim _{\Gamma_{\delta} \ni t \rightarrow 0} f(., t)$ exists in $\mathcal{D}^{\prime}\left(B_{r}(0)\right)$.

Proof. Write $Z(x, t)=\left(Z_{1}(x, t), \ldots, Z_{m}(x, t)\right), x=\left(x_{1}, \ldots, x_{m}\right), t=\left(t_{1}, \ldots, t_{n}\right)$, and $A(x, t)=\left(\psi_{i j}(x, t)\right)_{1 \leq i \leq m, 1 \leq j \leq n}$ so that

$$
Z(x, t)=x+A(x, t) t .
$$

Since $\mathcal{V}$ is CR in $U, \Im A(x, t)$ has rank $n$ at and hence near the origin. Without loss of generality, suppose that

$$
B(x, t)=\left(\Im \psi_{i j}(x, t)\right)_{1 \leq i, j \leq n} \text { is invertible near the origin. }
$$

Then

$$
|A(x, t) t| \geq|B(x, t) t| \geq\left|B_{l}(x, t) \cdot t\right| \text { for all }(x, t) \text { near }(0,0),
$$

where $B_{l}(x, t)$ is the $l$-th row of $B(x, t)$. Fix $t^{0} \in \Gamma$. Since $B(0,0)$ is invertible, one can find a row $B_{l}(0,0)$ of $B(0,0)$ such that

$$
\left|B_{l}(0,0) \cdot \frac{t^{0}}{\left|t^{0}\right|}\right|=C_{0}>0
$$


Hence, we can find an open convex cone $\widetilde{\Gamma} \subset \subset \Gamma$ containing $t^{0}$ such that

$$
\left|B_{l}(0,0) \cdot \frac{t}{|t|}\right| \geq \frac{1}{2} C_{0} \text { for all } t \in \widetilde{\Gamma} .
$$

Therefore, we can find a wedge $\widetilde{\mathcal{W}}=B_{\widetilde{r}}(0) \times \widetilde{\Gamma}_{\delta} \subset \subset \mathcal{W}$ (where $0<\widetilde{r}<r$ ) such that

$$
\left|B_{l}(x, t) \cdot \frac{t}{|t|}\right| \geq \frac{1}{4} C_{0} \text { for all }(x, t) \in \widetilde{\mathcal{W}} .
$$

This implies that for all $(x, t) \in \widetilde{\mathcal{W}}$

$$
|Z(x, t)-Z(x, 0)|=|A(x, t) t| \geq \frac{1}{4} C_{0}|t| .
$$

Thus,

$$
|f(x, t)||t|^{N} \leq \text { const. }|f(x, t)||Z(x, t)-Z(x, 0)|^{N} \leq C .
$$

Hence, by Theorem 4.1, bf $=\lim _{\Gamma_{\delta} \ni t \rightarrow 0} f(., t)$ exists in $\mathcal{D}^{\prime}\left(B_{r}(0)\right)$.

\section{REFERENCES}

[A] C. H. Asano, On the $C^{\infty}$ Wave-front set of Solutions of First Order Nonlinear PDEs, Proc. of AMS, 123:10 (1995), pp. 3009-3019.

[BCT] M. S. Baouendi, C. H. Chang, And F. Treves, Microlocal hypo-analyticity and extension of CR functions, J. Differential Geom., 18 (1983), pp. 331-391.

[BCH] S. Berhanu, P. Cordaro, and J. Hounie, An Introduction to Involutive Structures, (2006), to appear in Cambridge University Press.

[BH1] S. Berhanu and J. Hounie, An F. and M. Riesz theorem for planar vector fields, Math. Ann., 320 (2001), pp. 463-485

[BH2] S. Berhanu And J. Hounie, Traces and the F. and M. Riesz Theorem for vector fields, Ann. Inst. Fourier, Grenoble, 53:5 (2003), pp. 1425-1460.

[EG] M. G. Eastwood and C. R. Graham, Edge of the wedge theory in hypo-analytic manifolds, Commun. Partial Differ. Equations, 28 (2003), pp. 2003-2028.

[T] F. Treves, Hypo-analytic structures, Princeton University Press, (1992). 
Z. ADWAN AND S. BERHANU 